\title{
MicroRNA signatures from multidrug-resistant Mycobacterium tuberculosis
}

\author{
NA REN ${ }^{1}$, GUIJU GAO ${ }^{1}$, YUE SUN ${ }^{2}$, LING ZHANG $^{1}$, HUIZHU WANG $^{1}$, \\ WENHAO HUA $^{1}$, KANGLIN WAN ${ }^{3}$ and XINGWANG LI ${ }^{1}$
}

\begin{abstract}
${ }^{1}$ The National Clinical Key Department of Infectious Diseases, Beijing Ditan Hospital, Capital Medical University, Beijing 100015; ${ }^{2}$ Department of Infectious Diseases, First Hospital of Tsinghua University, Beijing 100016;

${ }^{3}$ National Institute for Communicable Disease Control and Prevention, Chinese Center for Disease Control and Prevention, State Key Laboratory for Infectious Disease Prevention and Control, Beijing 102206, P.R. China
\end{abstract}

Received July 2, 2014; Accepted April 10, 2015

DOI: $10.3892 / \mathrm{mmr} .2015 .4262$

\begin{abstract}
Tuberculosis (TB) infections, caused by multidrug-resistant Mycobacterium tuberculosis (MDR MTB), remain a significant public health concern worldwide. The regulatory mechanisms underlying the emergence of MDR MTB strains remain to be fully elucidated, and further investigation is required in order to develop better strategies for TB control. The present study investigated the expression profile of microRNA (miRNA) in MTB strains, and examined the differences between sensitive MTB and MDR MTB using next generation sequencing (NGS) with Illumina Deep Sequencing technology to better understand the mechanisms of resistance in MDR MTB, A total of 5, 785 and 195, and 6, 290 and 595 qualified Illumina reads were obtained from two MDR MTB strains, and 6, 673 and 665, and 7, 210 and 217 qualified Illumina reads were obtained from two sensitive MTB strains. The overall de novo assembly of miRNA sequence data generated 62 and 62, and 95 and 112 miRNAs between the 18 and 30 bp long from sensitive MTB strains and MDR MTB strains, respectively. Comparative miRNA analysis revealed that 142 miRNAs were differentially expressed in the MDR MTB strain, compared with the sensitive MTB strain, of which 48 were upregulated and 94 were downregulated. There were six similarly expressed miRNAs between the MDR and
\end{abstract}

Correspondence to: Professor Xingwang Li, The National Clinical Key Department of Infectious Diseases, Beijing Ditan Hospital, Capital Medical University, 8 Jingshun East, Beijing 100015, P.R. China

E-mail: dtyygr@163.com

Professor Kanglin Wan, National Institute for Communicable Disease Control and Prevention, Chinese Center for Disease Control and Prevention, State Key Laboratory for Infectious Disease Prevention and Control, Beijing 102206, P.R. China

E-mail: wankanglin@icdc.cn

Key words: microRNA, multi-drug-resistant, next generation sequencing, Mycobacterium tuberculosis sensitive MTB strains, and 108 miRNAs were expressed only in the MDR MTB strain. The present study acquired miRNA data from sensitive MTB and MDR MTB strains using NGS techniques, and this identification miRNAs may serve as an invaluable resource for revealing the molecular basis of the regulation of expression associated with the mechanism of drug-resistance in MTB.

\section{Introduction}

Tuberculosis (TB) is one of the most life-threatening infectious diseases worldwide, second only to human immunodeficiency virus. An estimated 2 billion individuals are infected with the bacteria that cause TB, and each year 8 million individuals are diagnosed with the disease (1). Despite therapy, the mortality rate of TB between $\sim 2$ and 3 billion annually (1). Adding to the considerable burden of TB-associated morbidity and mortality rates are drug-resistant strains of the disease. Multidrug-resistant (MDR) TB (MDR MTB) is a strain of Mycobacterium tuberculosis, which expresses in vitro resistance to at least rifampicin and isoniazid, which are two of the most powerful antituberculosis agents available. Resistance to these drugs results in longer, more complicated and costly treatment for TB (1). Developing countries account for $95 \%$ of all TB cases and $98 \%$ of all TB-associated mortality worldwide (2). Of these, $>12 \%$ are diagnosed with MDR MTB (1).

The distinctive features of Mycobacterium tuberculosis, including slow growth rate, dormancy, unique cell wall composition and resistance towards phagocytosis by macrophages, require detailed investigation at the molecular level (3). A number of previous studies have revealed significant differences in the transcription process of mycobacteria, compared with Escherichia coli and other bacteria $(4,5)$.

MicroRNAs (miRNAs) are a subset of non-coding RNAs, $\sim 22$ nucleotide (nt) long, which post-transcriptionally regulate gene expression by base-pairing with target mRNAs. miRNAs are transcribed as pri-miRNAs in the nucleus and are then processed into pre-miRNAs. Following translocation to the cytoplasm, a mature $22 \mathrm{nt}$ duplex is formed. One miRNA strand is then incorporated into the RNA-induced silencing complex, and interacts with its target mRNA via base-pairing 
at binding sites, usually located within 3' untranslated regions, whereas the other strand is usually degraded (6). Depending on the level of miRNA-mRNA complementarity, the target mRNA is either degraded or its translation is repressed (7).

miRNAs constitute an evolutionarily conserved system, which is associated with the regulation of biological functions at the post-transcriptional level. The capability of organisms to rapidly adapt their metabolism is essential for survival, and miRNAs are used by cells to rapidly transfer and internalize an external signal (8). Furthermore, in addition to affecting translation, miRNAs can affect epigenetic processes. miRNAs have been identified in almost all kingdoms of life, including archaea, humans and plants (8). However, the way in which miRNA regulates the expression of mRNA at the translational level in TB remains to be elucidated. The present study analyzed differences between the miRNA expression profiles of MDR MTB and drug-sensitive TB, in order to identify novel mRNA transcripts associated with drug resistance in $\mathrm{TB}$.

\section{Materials and methods}

Mycobacterium tuberculosis strains. Mycobacterium tuberculosis strains were isolated from lung tissue specimens of four patients, hospitalized with TB, diagnosed at the Beijing Ditan Hospital (Beijing, China), between April 2010 and October 2012. Lung tissue was obtained by thoracoscopic lung biopsy. TB was diagnosed based on the Chinese Pulmonary Tuberculosis Diagnostic Criteria (WS288-2008) and the Chinese TB Volume of Clinical Diagnosis and Treatment Guidelines (9).

The present study was approved by the Ethics Committee of Beijing Ditan Hospital, Capital Medical University (Beijing, China), according to the Declaration of Helsinki (10). Written informed consent was obtained from all of the patients.

Culture and identification of Mycobacterium tuberculosis. Sputum/bronchoalveolar lavage fluid, pleural fluid and tissue samples were collected from the patients with TB for mycobacterial assessment, by culturing the bacteria with either Lowenstein-Jensen (L-J) culture medium (Roche Molecular Systems, Inc., Branchburg, NJ, USA) or in a BACTEC 960 system (Roche Molecular Systems, Inc.). Blood and sputum samples, were initially cultured using the BACTEC 9120 Blood Culture system (Roche Molecular Systems, Inc.), and the positive samples were then cultured in L-J media at $37^{\circ} \mathrm{C}$ for 30 days. The Mycobacterium strains were identified using multi-locus polymerase chain reaction (PCR; Roche Molecular Systems, Inc.) $(11,12)$. The total volume of the PCR reaction mixture was $25 \mu 1$ (13 $\mu 1$ 2X PCR mix; $1 \mu 1$ forward primer; $1 \mu 1$ reverse primer; $1 \mu 1$ DNA template; $9 \mu \mathrm{l}$ DNase free water). The PCR amplification procedure was as follows: Pre-degeneration at $94^{\circ} \mathrm{C}$ for $5 \mathrm{~min}$; 35 cycles of degeneration at $94^{\circ} \mathrm{C}$ for $1 \mathrm{~min}$, annealing at $60^{\circ} \mathrm{C}$ for $1 \mathrm{~min}$ and extension at $72^{\circ} \mathrm{C}$ for $1 \mathrm{~min}$; followed by a final extension step at $72^{\circ} \mathrm{C}$ for $10 \mathrm{~min}$. The Mastercycler Nexus (Eppendorf, Hamburg, Germany) was used to conduct PCR. The sequences of the primers (synthesized by Invitrogen Life Technologies, Carlsbad, CA, USA) were as follows (lower case, plasmid; upper case, TB): Forward (F)1, tgt aaacgacg gccagtCGGATMACCGCTTTCGCCG, reverse (R)1, caggaacagctatgaccGACATGTGTGAGCTGTTT GC; F2, tgtaaaacgacggccagtGAAGGCGGTATTCAAGC, R2, caggaaacagctatgaccGAGTCACCCTCCACAATGTA;F3, tgtaaacgacggccagtGAAACCATTTCAACGGGTTC, R3, caggaaacagctatgaccCCATTGTAGCTGTACCAAGCACCC; F4, tgtaaaacgacggccagtTGGCCATAACGACATTCTG, R4, caggaacagctatgaccGAGCACCAACGTGTTTAGC; F5, tgtaaacgacggccagtACGGCTACGCAAAAGAAATG; R5, caggaacagctatgaccTTGAGGCTGAGCCGATACTT; F6, tgtaaacgacggccagtAGCAACCGGTAAAATTGTCG, R6, caggaaacagctatgaccCAGTGTAAGAACCGGCACAA; and F7, tgtaaacgacggccagtTGTACGAAATTGCCACCAAA, and R7, caggaaacagctatgaccAATATTTTCGCCGCATCAAC. Drug sensitivity testing (DST) of the Mycobacterium tuberculosis strains was performed using the proportion method with four first-line anti-tuberculosis drugs: Isoniazid, rifampicin, streptomycin and ethambutol. Briefly, the tested bacteria liquid $(20 \mathrm{mg} / \mathrm{l})$ was prepared, and then $0.01 \mathrm{ml}$ bacteria liquid was inoculated in L-J medium, with was $0.1 \mu \mathrm{g}$ and $0.001 \mu \mathrm{g}$ of each drug, respectively. After inoculation in $37^{\circ} \mathrm{C}$ culture medium for 4 weeks, the colony numbers were counted. If the percentage of colony numbers compared to control is $\leq 1 \%$ the strain is considered sensitive, and if it is $>1 \%$ the strain is considered resistant.

RNA extraction. RNA was extracted from the Mycobacterium tuberculosis using the RNA isolation reagent TRIzol ${ }^{\circledR}$ (Invitrogen Life Technologies). Initially, $30 \mathrm{mg}$ cultured Mycobacterium tuberculosis was added to a methanol/chloroform (Sigma-Aldrich, St. Louis, MO, USA) suspension (1:3). The suspension was then agitated with $5 \mathrm{ml}$ $\mathrm{TRIzol}^{\circledR}$, centrifuged at $1,000 \mathrm{x} \mathrm{g}$ for $15 \mathrm{~min}$ at $4^{\circ} \mathrm{C}$, and the colorless upper phase was collected. An equivalent quantity of isopropanol $(0.5 \mathrm{ml})$ was mixed with this upper phase, centrifuged and the collected sediment at the bottom of the tube was mixed with $\mathrm{dd}_{2} \mathrm{O}$. To prevent DNA contamination, the total RNA was treated with $20 \mu 1$ RNase-free DNase II (Invitrogen Life Technologies).

Next-generation sequencing (NGS). Small RNA fractions, with a length $\leq 50 \mathrm{nt}$, were subjected to hybridization and ligation using Adaptor mix (Agilent Technologies, Santa Clara, CA, USA). Subsequently, the RNA samples were reverse transcribed and sequenced using miRNA sequencing on an Illumina HiSeq 2000 platform (Illumina, San Diego, CA, USA).

The total RNA was isolated from each sample using TRIzol $^{\circledR}$, and the degradation and contamination of RNA was assessed using agarose gel (Abcam, Cambridge, MA, USA) electrophoresis. The RNA was purified using a KingFisher ${ }^{\mathrm{TM}}$ Pure RNA Tissue kit (Thermo Fisher Scientific, Inc., Waltham, MA, USA), and the ratio of the absorbance at 260 and $280 \mathrm{~nm}$ (A260/280) was determined to confirm purity. The RNA integrity was assessed using an RNA Nano 6000 Assay kit on a Bioanalyzer 2100 system (Agilent Technologies). A total of $3 \mathrm{~g}$ RNA per sample was used as input material for the RNA sample preparations, and all samples had RNA integrity number values $>8$. The samples of three individuals were then pooled within each group in equal quantities to 
generate two mixed samples. The pooled samples were then used to prepare six separate Illumina sequencing libraries, containing three technical replicates for each condition. cDNA libraries were generated using an Illumina TruSeq ${ }^{\mathrm{TM}}$ RNA Sample Preparation kit (Illumina), according to the manufacturer's instructions, and index codes were added to attribute sequences to each sample. Briefly, the mRNA was purified from the total RNA using poly-T oligo-attached magnetic beads (Thermo Fisher Scientific, Inc., Rockford, IL, USA). Fragmentation was performed using divalent cations under elevated temperature $\left(55^{\circ} \mathrm{C}\right)$ in Illumina proprietary fragmentation buffer (Illumina). First strand cDNA was synthesized using random oligonucleotides and SuperScript II (Abcam). Second strand cDNA synthesis was subsequently performed using DNA Polymerase I and RNase H (Abcam). The remaining overhangs were converted into blunt ends via exonuclease/polymerase activities and the enzymes were removed. Following adenylation of the 3 ' ends of the DNA fragments, the Illumina PE adapter oligonucleotides were ligated to prepare for hybridization. In order to select cDNA fragments of $200 \mathrm{bp}$ in length, the library fragments were purified using an AMPure XP system (Beckman Coulter Genomics, Danvers, MA, USA). DNA fragments with ligated adaptor molecules on each end were selectively enriched using Illumina PCR Primer Cocktail (Illumina) in a 10 cycle PCR reaction. Primersequences were as follows: Forward: 5'-AATGATACGGCGACCACCGAGA-3' and reverse: 5'-CAAGCAGAAGACGGCATACGAGT-3'). Products were purified using AMPure XP system (Beckman Coulter, Beverly, MA, USA) and quantified using the Agilent high sensitivity DNA assay on the Agilent Bioanalyzer 2100 system (Agilent Technologies). Prior to sequencing, all the individual libraries were normalized and pooled together in a single lane on an Illumina HiSeq 2000 platform, and 0-100 bp paired-end reads were generated.

Data extraction and analysis. To detect standard Mycobacterium tuberculosis strains in PubMed, particularly CDC1551 (http://www.ncbi.nlm.nih.gov/Taxonomy/ Browser/wwwtax.cgi?id=83331), a library of reference sequences was prepared by identifying the sequences of mature miRNAs, together with five flanking nucleotides, within the hairpins deposited in miRBase version 19 (http://www.mirbase.org/) (13). The Illumina HiSeq platform requires a minimum read length of $50 \mathrm{nt}$, therefore, all small RNAs were extended using specific adapters (forward, 5'-AATGATACGGCGACCACCGAGA-3'; reverse, 5'-CAAGCAGAAGACGGCATACGAGT-3'), which were annealed to their $3^{\prime}$ ends during library preparation. Removal of the adapters was performed in silico on the raw, 50 nt Illumina sequence reads, using Cutadapt software (14). The resulting 15-30 nt long sequences were subjected to further analysis as potential miRNAs. The sequences were mapped on the prepared reference library using Bowtie version 0.12 .7 (15), with the requirement of perfect matching. The numbers of mapped reads were subsequently calculated for each miRNA and provided as a number of each of the unique reads mapped to each reference sequence, and as a number of all the reads mapped to each reference sequence. Data obtained for each sample was normalized using reads
Table I. Capacities of Illumina sequencing of MTB strains.

Strain

Number of reads

MDR MTB1

$5,785,195$

MDR MTB2

$6,290,595$

Sensitive MTB1

$6,673,665$

Sensitive MTB2

$7,210,217$

MDR, multi-drug resistant; MTB, Mycobacterium tuberculosis.

Table II. Quality of Illumina sequencing of MTB strains.

\begin{tabular}{lcc}
\hline Strain & Q20 $(\%)$ & Q30 (\%) \\
\hline MDR MTB1 & 99.56 & 91.50 \\
MDR MTB 2 & 99.65 & 91.93 \\
Sensitive MTB1 & 99.32 & 90.11 \\
Sensitive MTB2 & 99.45 & 90.62 \\
\hline
\end{tabular}

MDR, multi-drug resistant; MTB, Mycobacterium tuberculosis; Q20, error rate $=1 \% ; \mathrm{Q} 30$, error rate $=0.1 \%$.

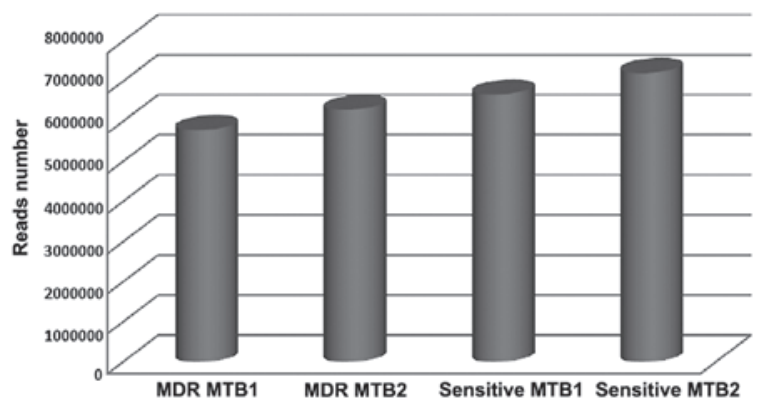

Figure 1. Capacity of sequencing. A total of 5, 785 and 195, and 6, 290 and 595 qualified Illumina reads were obtained from two MDR MTB strains. and 6, 673 and 665, and 7, 210 and 217 were obtained from two sensitive MTB strains. MDR, multi-drug resistant; MTB, Mycobacterium tuberculosis.

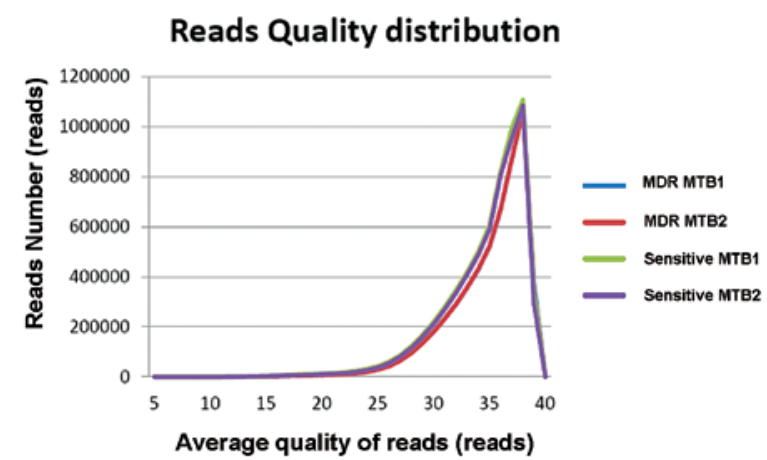

Figure 2. Quality of sequencing. According to the statistical results, the average quality of $>99 \%$ of reads was $>20$ in each sample. MDR, multi-drug resistant; MTB, Mycobacterium tuberculosis.

per million (RPM) normalization, according to the following formula: $\mathrm{RPM}=\left(\mathrm{N}_{\mathrm{ref}} / \mathrm{N}_{\mathrm{all}}\right) \times 10^{6} . \mathrm{N}_{\text {ref }}$ indicates the number of 
Table III. Data pre-processing of next generation sequencing.

\begin{tabular}{|c|c|c|c|c|c|}
\hline Strain & $\begin{array}{c}\text { Trim } 3^{\prime} \text { adapter } \\
\mathrm{n}(\%)\end{array}$ & $\begin{array}{c}\text { Filter low quality } \\
\mathrm{n}(\%)\end{array}$ & $\begin{array}{l}\text { Collapse tags } \\
\text { (n) }\end{array}$ & $\begin{array}{c}18-30 \mathrm{nt} \text { reads } \\
\mathrm{n}(\%)\end{array}$ & $\begin{array}{c}\text { 18-30 nt tags } \\
\text { (n) }\end{array}$ \\
\hline MDR MTB1 & $5,445,431(94.13)$ & $4,665,152(80.64)$ & $2,494,091$ & $2,583,186(44.65)$ & $1,438,171$ \\
\hline MDR MTB2 & $5,378,966(85.51)$ & $4,661,476(74.10)$ & $2,497,989$ & $2,829,821(44.98)$ & $1,552,250$ \\
\hline Sensitive MTB1 & $6,231,203(93.37)$ & $5,263,218(78.87)$ & $1,880,645$ & $2,541,061(38.08)$ & $1,056,595$ \\
\hline Sensitive MTB2 & $5,992,842(83.12)$ & $5,120,235(71.01)$ & $1,858,932$ & $2,766,439(38.37)$ & $1,125,144$ \\
\hline
\end{tabular}

MDR, multi-drug resistant; MTB, Mycobacterium tuberculosis; nt, nucleotides.

Table IV. Genome mapped tags and reads.

\begin{tabular}{lcc}
\hline Strain & $\begin{array}{c}\text { Genome-mapped } \\
\text { tags [n (\%)] }\end{array}$ & $\begin{array}{c}\text { Genome-mapped } \\
\text { reads [n (\%)] }\end{array}$ \\
\hline MDR MTB1 & $517,409(35.98)$ & $1,490,704(57.71)$ \\
MDR MTB2 & $554,600(35.73)$ & $1,637,876(57.88)$ \\
Sensitive MTB1 & $557,934(52.80)$ & $1,836,536(72.27)$ \\
Sensitive MTB2 & $590,434(52.48)$ & $2,002,245(72.38)$ \\
\hline
\end{tabular}

MDR, multi-drug resistant; MTB, Mycobacterium tuberculosis.

Reads Length Distribution

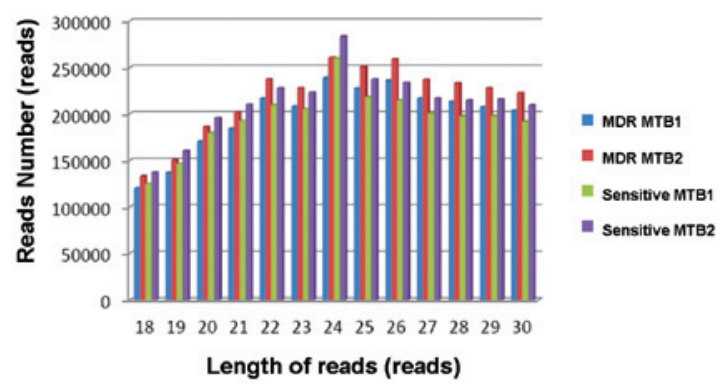

Figure 3. Read length distribution. The raw data of next generation sequencing are presented. MDR, multi-drug resistant; MTB, Mycobacterium tuberculosis.

reads mapped to the miRNA reference, and $\mathrm{N}_{\text {all }}$ indicates the total number of reads mapped in the sample (16).

Statistical analysis. Selection of miRNAs and isomiRs deregulated between the analyzed groups was performed using a Welch t-test, paired for comparison between sensitive MTB and MDR MTB samples. False discovery rate was used to assess multiple testing errors. Statistical analyses were conducted using SPSS version 13.0 (SPSS Inc., Chicago, IL, USA) Hierarchical clustering of samples, based on the expression profiles of the selected miRNAs, was performed using Ward's agglomeration method, operated on Euclidean distance measures. Identification of target genes for each miRNA seed sequence, with significantly deregulated expression between the isoniazid-sensitive MTB and MDR MTB samples was performed using Target Rank version 3.2 software $(16,17)$.
sRNA Length Distribution

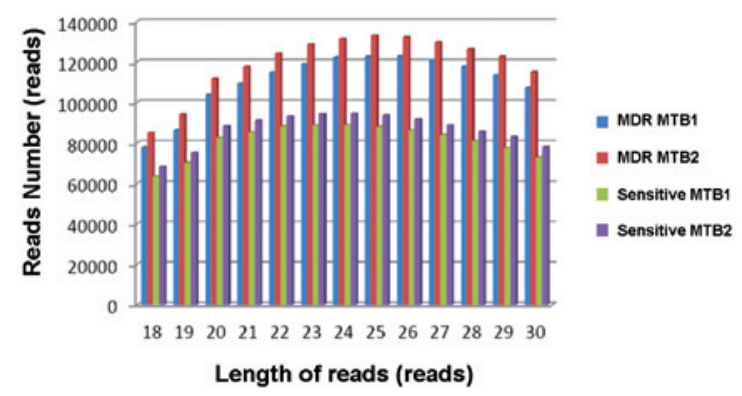

Figure 4. sRNA length distribution. The sRNA tags are shown in the graph. MDR, multi-drug resistant; MTB, Mycobacterium tuberculosis; sRNA, small RNA.

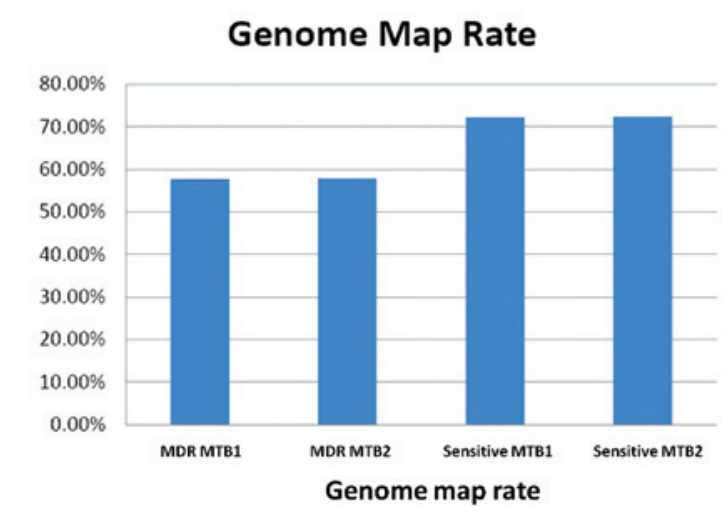

Figure 5. Genome map rate. The graph displays the genome map rate of the MTB samples. MDR, multi-drug resistant; MTB, Mycobacterium tuberculosis.

\section{Results}

Capacity and quality of sequencing. A total of 5, 785 and 195, and 6, 290 and 595 qualified Illumina reads were obtained from the two MDR MTB strains; and 6, 673 and 665, and 7, 210 and 217 qualified Illumina reads were obtained from the two sensitive MTB strains, respectively (Table I and Fig. 1). According to the statistical results, the average quality of $>99 \%$ of the reads was $>20$ in each sample, indicating the quality of the sequencing was suitable (Table II and Fig. 2).

Data pre-processing of NGS. The adapter sequence (3' adapter: AGATCGGAAGAGCACACGTCT) was filtered from the 
Table V. Reads from Rfam analysis.

\begin{tabular}{|c|c|c|c|c|}
\hline Data & MDR MTB1 & MDR MTB2 & Sensitive MTB1 & Sensitive MTB2 \\
\hline rRNA & 161,738 & 176,797 & 208,101 & 226,316 \\
\hline Other RNA & 25,089 & 27,441 & 26,246 & 28,405 \\
\hline tRNA & 23,992 & 26,474 & 80,832 & 88,883 \\
\hline $\operatorname{lncRNA}$ & 98 & 83 & 136 & 130 \\
\hline sRNA & 632 & 680 & 2,411 & 2,643 \\
\hline snRNA & 309 & 292 & 367 & 417 \\
\hline Genes & 710,982 & 779,884 & 932,552 & $1,016,764$ \\
\hline Total reads & 922,840 & $1,011,651$ & $1,250,645$ & $1,363,588$ \\
\hline Percentage & 61.91 & 61.77 & 68.10 & 68.10 \\
\hline
\end{tabular}

Percentage refers to the percentage of detected gene reads out of the total reads. MDR, multi-drug resistant; MTB, Mycobacterium tuberculosis.

Table VI. Tags from Rfam analysis.

\begin{tabular}{lcccc}
\hline Data & MDR MTB1 & MDR MTB 2 & Sensitive MTB1 & Sensitive MTB2 \\
\hline rRNA & 10,425 & 10,708 & 10,270 & 10,387 \\
Other RNA & 3,965 & 4,127 & 4,259 & 4,425 \\
tRNA & 1,963 & 2,050 & 2,310 & 2,434 \\
lncRNA & 55 & 44 & 61 & 66 \\
sRNA & 264 & 256 & 365 & 270 \\
snRNA & 228 & 216 & 223 & 478,639 \\
Genes & 410,107 & 440,968 & 450,926 & 468,414 \\
Total reads & 427,007 & 458,369 & 83.96 & 84.10 \\
Percentage & 82.53 & 82.65 & 878 \\
\hline
\end{tabular}

Percentage refers to the percentage of detected gene reads out of the total reads. MDR, multi-drug resistant; MTB, Mycobacterium tuberculosis.

Table VII. miRNAs from Mirdeep analysis.

\begin{tabular}{lcccc}
\hline Data & MDR MTB1 & MDR MTB 2 & Sensitive MTB1 & Sensitive MTB2 \\
\hline miRNA (n) & 62 & 62 & 95 & 112 \\
miRNA reads (n) & 33,051 & 36,243 & 75,015 & 81,954 \\
Percentage & 2.22 & 2.21 & 4.08 & 4.09 \\
miRNA tags (n) & 2,606 & 2,756 & 3,627 & 4,024 \\
Percentage & 0.50 & 0.50 & 0.65 & 80.68
\end{tabular}

Percentage refers to the percentage of detected gene reads out of the total reads. miRNA, microRNA; MDR, multi-drug resistant; MTB, Mycobacterium tuberculosis.

NGS raw data (Fig. 3) using the fastx_cliper program. The low quality reads were removed using the fastq_quality_filter program (http://seqanswers.com/forums/showthread. php?goto=nextoldest $\& \mathrm{t}=24679$ ), to ensure the quality score of at least $95 \%$ of the bases was $>20$. Clustering was then performed and identical base sequences were recorded as one tag. The length of the mature miRNA sequences was between 18 and $30 \mathrm{nt}$, which were further analyzed (Table III). The tags are shown in Fig. 4.
Analysis of small RNA. The tags were mapped to the genome sequence of the Mycobacterium tuberculosis strain CDC1551 in PubMed, using match software Bowtie version 0.12.7. The genome-mapped tags and reads, which were calculated based on 18-30 nt reads or tags, are shown in Table IV. The genome map rates of the samples are shown in Fig. 5. The RNA family database, Rfam (version 11.0; http://rfam.xfam.org/) was used to analyze the variety of RNAs in the samples. The Rfam reads and tags are shown in Tables V and VI. 
Analysis of miRNAs. Mirdeep (version 2; http://www. mdc-berlin.de/8551903/en/research/research_teams/systems_ biology_of_gene_regulatory_elements/projects/miRDeep) was used to predict the miRNAs in the samples. The data are shown in Table VII. The target genes of miRNAs were predicted using Miranda software (http://www.miranda-im. org/; score $>150$; energy $<-15$ ).

\section{Discussion}

There are increasing cases of MDR MTB, which do not respond to the existing first-line anti-TB drugs, including rifampicin, and isoniazid. In addition, extremely drug resistant (XDR) TB strains do not respond to even the most effective second-line anti-TB drugs (18-23). Therefore, there is an urgent requirement to identify the drug resistance mechanisms of MTB, and develop anti-TB drugs, which are effective against MDR and XDR TB strains. Bacterial RNA polymerase (RNAP) is the target for the anti-TB drug, rifampicin (24-26). Therefore, RNAP is considered an attractive target for the development of novel anti-TB drugs (27-30). To identify the drug resistance mechanisms of $\mathrm{MTB}$, and screen anti-TB agents, a high-throughput in vitro transcription and transcript regulation assay is required.

The demand for low-cost sequencing has driven the development of high-throughput sequencing, also termed NGS; however, NGS is only modestly accurate at absolute quantification. Thousands or millions of sequences are concurrently produced in the NGS process. Genome-wide computational analysis is increasingly being used for novel discoveries in biomedical research. However, as the quantities of sequence data increase exponentially, an analysis bottle-neck remains (31).

The present study performed a miRNA sequence analysis of sensitive MTB and MDR MTB using NGS. A total of 5, 785 and 195, and 6, 290 and 595 qualified Illumina reads were obtained from two MDR MTB strains, and 6,673 and 665, and 7,210 and 217 qualified Illumina reads were obtained from two sensitive MTB strains. The quality of the data was considered to be high, with the average quality of $>99 \%$ of reads being $>20$. Data pre-processing of NGS was performed using the fastx_cliper program, and low quality reads were removed using the fastq_quality_filter program. Clustering was performed, and the mature miRNA sequences between 18 and $30 \mathrm{nt}$, were mapped to the genome sequence of the Mycobacterium tuberculosis strains. The target genes of the miRNAs were then predicted using Miranda software (score $>150$; energy $<-15$ ).

Rfam is a collection of multiple sequence alignments and covariance models, representing non-coding RNA families. Rfam is available online (http://rfam.sanger.ac.uk/; http://rfam.janelia.org/), and allows users to search a query sequence against a library of covariance models, and view multiple sequence alignments and family annotation. The database can also be downloaded in flatfile form and searched locally, using the INFERNAL package (http://infernal.wustl. edu/). The first analysis by Rfam contains 25 families, which annotate $>50,000$ non-coding RNA genes in the taxonomic divisions of the EMBL nucleotide database (http://www.ebi. ac.uk/embl).
The overall de novo assembly of miRNA sequence data generated 62 and 62, and 95 and 112 miRNAs with a length of 18-30 bp from the MDR MTB and sensitive MTB strains, respectively. Comparative miRNA analysis identified 142 differentially expressed miRNAs in the MDR MTB strain, compared with the sensitive MTB strain, and included 48 upregulated and 94 downregulated genes. There were 108 miRNAs expressed only in MDR MTB.

miRNAs are short, non-coding RNAs, which bind to complementary sequences in the $3^{\prime}$ untranslated regions of protein-coding genes and regulate their expression $(32,33)$. Aberrant expression of miRNAs results in aberrant expression of their target mRNAs. A single miRNA regulates the expression of numerous genes (34), and several miRNAs may concurrently regulate the expression of a single gene, the function of which is pivotal in a given tissue (35). The recognition of mRNA by miRNA depends on the 'seed region' of a miRNA, comprising 2-8 nt of a mature molecule (17).

The majority of previous studies on miRNA aberrations were based on the analysis of the expression of canonical, reference miRNAs, as the analysis of isomiRs requires the use of more robust technologies, including NGS, and extensive analysis of the obtained data. Therefore, the present study used NGS to identify all the miRNA isoforms, which may be expressed in sensitive MTB or MDR MTB, the aberrances of which potentially underlie the initiation and progression of drug resistance. The aim of the present study was to identify novel, previously unknown isomiRs, and the results revealed the expression profiles of canonical miRNAs and their newly identified isoforms.

In conclusion, the present study demonstrated that miRNAs may serve as an invaluable resource for revealing the molecular basis of the regulation of expression associated with the mechanism of drug resistance in MTB.The differential expression of miRNAs between sensitive and MDR MTB was identified by NGS, and identified miRNAs that may be associated with the drug resistance of TB. However, the mechanism by which miRNA regulates the expression of mRNA at the translational level in TB remains to be elucidated. The present study analyzed the differences between the miRNA expression profiles of MDR MTB and drug-sensitive TB, in order to identify novel mRNA transcripts associated with drug resistance in $\mathrm{TB}$.

\section{Acknowledgements}

The present study was funded by the projects of the National Key Program of Mega Infectious Disease (grant nos. 2012ZX10005010-001, 2012ZX10005010-003 and 2013ZX10003002-001). The funding bodies had no role in the study design, data collection and analysis, manuscript preparation or decision to publish.

\section{References}

1. World Health Organization (WHO): Global Tuberculosis Control Report 2010. WHO, Geneva, 2010.

2. US Agency for International Development: Report to congress: Health-related research and development activities at USAID-an update on the five-year strategy, 2006-2010. US Agency for International Development 182: 1788-1790, 2009. 
3. Tare P, China A and Nagaraja V: Distinct and contrasting transcription initiation patterns at Mycobacterium tuberculosis promoters. PLoS One 7: e43900, 2012.

4. Jia $Y$ and Patel SS: Kinetic mechanism of transcription initiation by bacteriophage T7 RNA polymerase. Biochemistry 36: 4223-4232, 1997.

5. Martínez-Antonio A, Salgado H, Gama-Castro S, et al: Environmental conditions and transcriptional regulation in Escherichia coli: A physiological integrative approach. Biotechnol Bioeng 84: 743-749, 2003.

6. Holley CL and Topkara VK: An introduction to small non-coding RNAs: miRNA and snoRNA. Cardiovas Drugs Ther 25: 151-159, 2011.

7. Bartel DP: MicroRNAs: Target recognition and regulatory functions. Cell 136: 215-233, 2009.

8. Baroni D and Arrigo P: MicroRNA target and gene validation in viruses and bacteria. Methods Mol Biol 1107: 223-231, 2014.

9. Chinese Medical Association: TB volume of clinical diagnosis and treatment guidelines. People's Medical Publishing House Beijing, 2005.

10. World Medical Association: World Medical Association Declaration of Helsinki: Ethical principles for medical research involving human subjects. JAMA 310: 2191-2194, 2013.

11. Huard RC, Lazzarini LC, Butler WR, van Soolingen D and Ho JL: PCR-based method to differentiate the subspecies of the Mycobacterium tuberculosis complex on the basis of genomic deletions. J Clin Microbiol 41: 1637-1650, 2003.

12. Bao X, Lian L, Xu D, et al: Rapid species identification of 391 clinical Mycobacterium isolates from Anhui province by multi-locus PCR. Chinese Journal of Zoonoses 28: 659-663, 2012.

13. Kozomara A and Griffiths-Jones S: miRBase: Integrating microRNA annotation and deep-sequencing data. Nucleic Acids Res 39: D152-D157, 2011.

14. Martin M: Cutadapt removes adaptor sequences from high-throughput sequencing reads. EMBnet.journal 17: 10-12, 2011.

15. Langmead B, Trapnell C, Pop M and Salzberg SL: Ultrafast and memory-efficient alignment of short DNA sequences to the human genome. Genome Biol 10: R25, 2009.

16. Wojcicka A, Swierniak M, Kornasiewicz O, et al: Next generation sequencing reveals microRNA isoforms in liver cirrhosis and hepatocellular carcinoma. Int J Biochem Cell Biol 53: 208-217, 2014.

17. Nielsen CB, Shomron N, Sandberg R, Hornstein E, Kitzman J and Burge CB: Determinants of targeting by endogenous and exogenous microRNAs and siRNAs. RNA 13: 1894-1910, 2007.

18. World Health Organization (WHO): Global tuberculosis report, 2012. WHO, Geneva, 2012.

19. Dye C, Espinal MA, Watt CJ, Mbiaga C and Williams BG: Worldwide incidence of multidrug-resistant tuberculosis. J Infect Dis 185: 1197-1202, 2002.
20. Raviglione MC, Gupta R, Dye CM and Espinal MA: The burden of drug-resistant tuberculosis and mechanisms for its control. Ann NY Acad Sci 953: 88-97, 2001.

21. Zumia A and Grange JM: Multidrug-resistant tuberculosis - can the tide be turned? Lancet Infect Dis 1: 199-202, 2001.

22. World Health Organization (WHO): Anti-tuberculosis drug resistance in the world: The WHO/IUATLD global project on anti-tuberculosis drug resistance suveillance: Third global report. WHO, Geneva, 2003.

23. Banerjee R, Rudra P, Prajapati RK, Sengupta S and Mukhopadhyay J: Optimization of recombinant Mycobacterium tuberculosis RNA polymerase expression and purification. Tuberculosis (Edinb) 94: 397-404, 2014

24. Artsimovitch I, Vassylyeva MN, Svetlov D, et al: Allosteric modulation of the RNA polymerase catalytic reaction is an essential component of transcription control by rifamycins. Cell 122: 351-363, 2005.

25. Campbell EA, Korzheva N, Mustaev A, et al: Structural mechanism for rifampicin inhibition of bacterial RNA polymerase. Cell 104: 901-912, 2001

26. Feklistov A, Mekler V, Jiang Q, et al: Rifamycins do not function by allosteric modulation of binding of $\mathrm{Mg}^{2+}$ to the RNA polymerase active center. Proc Natl Acad Sci USA 105: 14820-14825, 2008.

27. Chopra I: Bacterial RNA polymerase: A promising target for the discovery of new antimicrobial agents. Curr Opin Investig Drugs 8: 600-607, 2007.

28. Chopra I, Hesse L and O'Neill AJ: Exploiting current understanding of antibiotic action for discovery of new drugs. Symp Ser Soc Appl Microbiol (31): 4S-15S, 2002.

29. Darst SA: New inhibitors targeting bacterial RNA polymerase. Trends Biochem Sci 29: 159-160, 2004.

30. Villain-Guillot P, Bastide L, Gualtieri M and Leonetti JP: Progress in targeting bacterial transcription. Drug Discov Today 12: 200-208, 2007.

31. Zhang J, Chiodini R, Badr A and Zhang G: The impact of next-generation sequencing on genomics. J Genet Genomics 38: 95-109, 2011.

32. Bartel DP: MicroRNAs: Target recognition and regulatory functions. Cell 136: 215-233, 2009.

33. Filipowicz W, Bhattacharyya SN and Sonenberg N: Mechanisms of post-transcriptional regulation by microRNAs: Are the answers in sight? Nat Rev Genet 9: 102-114, 2008.

34. Lim LP, Lau NC, Garrett-Engele P, et al: Microarray analysis shows that some microRNAs downregulate large numbers of target mRNAs. Nature 433: 769-773, 2005.

35. Jazdzewski K, Boguslawska J, Jendrzejewski J, et al: Thyroid hormone receptor beta (THRB) is a major target gene for microRNAs deregulated in papillary thyroid carcinoma (PTC). J Clin Endocrinol Metab 96: E546-E553, 2011 